\title{
The affective component of secure base schema in a Japanese and a Chinese sample: Evidence from affective priming paradigm ${ }^{1}$
}

\author{
Tong-gui $\mathrm{Li}^{2}$ \\ Department of psychology, Peking University, P. R. China
}

Kazuo Kato

Faculty of Human-Environment Studies, Kyushu University, Japan

\begin{abstract}
The affective priming paradigm (Murphy \& Zajonc, 1993) was adapted to assess the affective component of the secure base schema in a Japanese (Study 1) and a Chinese sample (Study 2). The priming effects of secure base pictures and positive pictures on evaluation of neutral stimuli were examined. The attachment dimensions, both anxiety and avoidance, were also measured for all participants. The subliminal priming of secure base pictures was found to lead to greater positive liking ratings to neutral stimuli than neutral priming pictures or no pictures in the Chinese but not in the Japanese sample. The results were discussed in the context of the dual-process theory of the secure base schema.
\end{abstract}

Keywords: Secure base schema, affective priming, adult attachment,

According to the attachment theory (Bowlby, 1969, 1973, 1980), internal working model of self and others formed in the context of the child-caregiver interaction can carry forward and influence thought, feeling and behavior in adult relationships (Pietromonaco \& Feldman Barrett, 2000). This study is designed to answer whether the secure base schema has a positive valence in adult attachment using an affective priming paradigm and whether there is any cultural difference between Japanese and

\footnotetext{
1 Author note: This research was supported by Natural Science Foundation of China (NSFC, No. 50978155), and Key Projects in the National Science \& Technology Pillar Program of China (No. 2009BAI77B04) to Tong-gui Li. We are indebted to Ai-Bao Zhou for running experiment in China. We thank Dazi Li and Shunichi Nogami for their help on writing computer program. We also thank Shima Wada for providing a Japan picture of Study 1, and Takako Otabe for recruiting subjects of Study 1 . We thank Takahiro Hisazaki and Tatsuma Nakao for their comments on an earlier draft of this paper. Correspondence concerning this paper should be addressed to Tong-gui Li, Department of Psychology, Peking University, Beijing, 100871, P. R. China, or Kazuo Kato, Department of Psychology, Faculty of Human-Environment Studies, Kyushu University, Fukuoka, 812-8581, Japan. Electronic mail may be sent to litg@pku.edu.cn or kkatoedu@mbox.nc.kyushu-u.ac.jp.

2 Address correspondence to Tong-gui Li (mail: litg@pku.edu.cn) or Kazuo Kato (mail: kkatoedu@ mbox.nc.kyushu-u.ac.jp).
} 
Chinese college students in the affective component of secure base schema (Murphy \& Zajonc, 1993; Murphy, Monahan \& Zajonc, 1995).

\section{Attachment research and secure base schema}

Attachment theory is based on the notion that human beings are innately programmed to seek and form close emotional bonds with the primary caregiver to boost survival advantage (Bowlby, 1969). Human infants possess a system of behaviors that has evolved to promote proximity to a caregiver (Ainsworth, 1989). With cognitive development, infants' experiences based on repeated interactions with the primary caregiver are internalized into their working models, which include some expectations about: (1) the caregiver's availability and responsiveness and (2) the infant's ability to elicit these behaviors from the caregiver. These "internal working models" (IWM) become integrated into the personality structure and influence individual's behavior (Lyddon, Bradford, \& Nelson, 1993). The knowledge that an attachment figure is available and responsive gives children a strong sense of "felt security". The attachment figure can be used as a "secure base from which to explore" (Waters \& Cummings, 2000).

Waters, Rodrigues, and Ridgeway (1998) hypothesized that the sense of felt security is organized into a basic prototype or script, termed 'secure base schema'. This script seems to include some rule-based processing mode (Smith et al., 2000), such as the if-then proposition: "If I encounter an obstacle and become distressed, I can approach a significant other for help; he or she is likely to be available and supportive; I will experience relief and comfort as a result of proximity to this person: I can then return to other activities" (Mikulincer, Hirschberger, Nachmias \& Gillath, 2001, p. 305).

We propose that the secure base schema also includes associative processing mode (Smith \& DeCoster, 2000), which can quickly and automatically provide information about previous experiences or affective reactions in situations that resemble the current one. The associative processing in secure base schema may be considered as knowledge of "how to be with someone" (Lyons-Ruth, 1998; Davis, 2001). It includes some implicit relational expectations, emotional responses, skills and habits that are elaborated and modified in important relationships during the life course, e.g., knowing how to express affection and how to get attention (Davis, 2001; Knox, 1999, 2001, 2003). It cannot be shared with others, and may never become symbolically coded. 
This hypothesis can be found in the original concept of internal working model (Bowlby, 1980), where the distinction between episodic and semantic memory system may imply that IWM of self and others are encoded during infancy at subsymbolic levels.

\section{Affective components of secure base schema}

During the last twenty years, several studies have examined the association between attachment security and emotional responses. Many studies have focused on conscious, rule-based processing, such as self-report (Hazan \& Shaver, 1987) and social diary method (Pietromonaco, \& Feldman Barrett, 1997). These studies consistently found that there are two adult attachment dimensions-- attachment anxiety and attachment avoidant (Brennan, et al., 1998), and the secure attachment style is related to positive affect (for a review see Fuendeling, 1998).

Although the association between positive affect and secure attachment style is consistently found, this may just reflect one side of the secure base schema, i.e. the rule-based processing mode, Fraley et al. (2000) consider also the term 'pre-emptive' and 'post-emptive' task. The associative processing mode aspect of the secure base schema may not be studied using the above methods. In fact, according to the dualprocess model in social psychology (Smith \& DeCoster, 2000), the features of associative processing mode and rule-based processing mode are different. For associative processing mode, it is quick and automatic, operating pre-consciously; information that has repeatedly been linked to an object in the past is automatically brought to mind. Associations are built up through repeated experiences over time and are not necessarily interpersonally shared or symbolically encoded.

How to assess the associative processing mode in the secure base schema? Previous researchers on the associative processing mode mainly have considered the clinical interview (AAI, Main, Kaplan \& Cassidy, 1985; Crittenden, 2000), case study (Carver, 2001; Knox, 2001) and behavior observation (Strange Situation, Ainsworth et al., 1978; Fraley \& Shaver, 1998). But these methods may not provide a direct measure of associative processing mode of secure base, because one does not know whether the secure base schema was indeed activated in those studies. With the recent development of cognitive psychology, researchers have used cognitive methods, such as lexical 
decision and affective priming, to study associative processing mode of attachment (e.g., Baldwin, et al., 1996; Banse, 1999, 2001; Mikulincer, et al., 2001).

The affective priming technique is likely the best way to examine the associative processing mode of secure base schema, because it reflects the "cognitive accessibility of positive affect, i.e., its readiness to use in mental activity" (Mikulincer, et al., 2001, p. 306). There is evidence that the mere activation of object representation can be sufficient to activate the associated affect (Fazio, Sanbonmatsu, Powell, \& Kardes, 1986). If the subject is unaware of the source of the affect activation, it can easily be misattributed to another object. According to Murphy, Zajonc and their colleagues (Murphy \& Zajonc 1993; Murphy, Monahan, \& Zajonc, 1995), affect can be brought to mind even before one recognizes it in conscious awareness and influence cognitive processes. Using priming technique, few studies found that the associative processing mode of secure base schema is also related to positive affect (Mikulincer, et al., 2001; Rowe \& Carnelley, 2003). For example, Mikulincer, et al., (2001) found that subjects place higher liking ratings for neutral Chinese characters when priming a secure base schema (Picasso's sketch) and a positive picture than a neutral picture (polygon). Thus, they concluded that a secure base schema imbues positive affect.

\section{Secure base schema and cultural difference}

As mentioned above, several studies demonstrated that associative processing mode of secure base schema per se imbues positive affect. But an emerging question is concerned with the universality of the association: Is there the same associative processing mode among different cultures? Given that attachment theory was proposed as a universal theory of human development, if attachment is biologically based and adaptive in the evolutionary sense, the major propositions in attachment theory should apply to all human beings across cultures (Colins, 1996; for review see van Ijzendoor \& Sagi, 1999).

But there are controversies on this topic. It is also true that child-rearing goals and caregiving patterns vary greatly from culture to culture, and may result in different emotional feelings. For example, it seems that there is a unique emotion in Japanese, known as amae, which cannot be identified in the Western country's emotion dimensions. Amae was low in activation, neither positive nor negative, fairly akin to sleepiness (Markus \& Kitayama, 1991, p.238). Moreover, Mizuta, Zahn-Waxler, Cole, 
\& Hiruma (1996) found that the Japanese preschoolers showed more amae behavior than US children. Vereijken, Hanta \& van Lieshout (1997) also found there was no relation of attachment security (i.e., to child-mother interaction or to peer interaction) in Japanese children using attachment Q-sort. Finally, Rothbaum and his colleagues (Rothbaum, Weisz, Pott, Miyake, Morelli, 2000; Rothbaum, Pott, Azuma, Miyake, \& Weisz, 2000; Rothbaum, Rosen, Ujiie, Uchida, 2002) argued that the Japanese might have associations between secure base and Amae behavior rather than with exploration behavior. Thus, their association in the secure base schema may not be the same as in western participants.

On the other hand, the subliminal priming technique has attracted several negative comments. Some of its critics have emphasized that subliminal priming effects are extremely difficult to replicate (Mayer \& Merckelbach, 1999). For example, the studies conducted in Japan found that there was no affective priming effect in Japanese subjects, using Murphy \& Zajonc (1993) procedure, irrespective of whether the target was seal engraving Chinese characters, Thailand characters, or ambiguity pictures (Ogawa, 1998).

In this article, we measure the associative processing mode in secure base schema of adult attachment in Japanese and Chinese college students, and explore possible cultural differences using Murphy \& Zajonc's (1993) affective priming paradigm.

\section{Study Hypotheses}

The main purpose of the study is to assess the affect component of associative processing mode in the secure base schema. To accomplish this goal, we use Picasso's sketch as a prime picture as in Mikulincer et al. (2001), because it contains the main features of attachment, and the priming effect was aroused by mother-infant caring interaction, instead of single picture of mother or baby (Mikulincer, et al., 2001, Study 2). A happy face is used as the positive prime picture, since facial configurations associated with happiness have been found to be universally recognized as indicators of positive affect, thus reducing the possibility of idiosyncratic responses to the primes (Murphy, Monahan \& Zajonc, 1995). At the same time, we use a polygon as a neutral prime picture, and a non-picture prime consisted of a blank gray background as baseline. Consistent with previous findings, we expect that the subliminal priming of secure base or positive prime pictures would produce higher liking ratings of the neutral 
target stimuli than neutral or non pictures would. We also predict that there is no significant difference between secure base prime and positive prime pictures.

Another factor relevant to the present study purposes is the notion of familiarity of association in adult attachment. In cognitive psychology, familiarity is defined as the quantitative strength of association (Yonelias, 2001). Stronger association leads to stronger familiarity. In order to measure familiarity, we also use an 'indigenous' picture, (a Japanese picture for Japanese subjects, a Chinese picture for Chinese subjects), which also contains a mother and a baby, as a secure base schema priming picture. This indigenous picture may be more familiar for subject than Picasso's sketch. Given that subjective familiarity will lead to higher positive affect (Smith, 2000, cited as Yamada, 2001), we expect that this indigenous prime picture will lead to a highest liking rating. Thus, our first hypothesis is that liking ratings for neutral targets will be ranked as: Indigenous picture $>$ Picasso picture $=$ Positive $>$ Neutral $=$ Non picture .

Several studies have found that there are no individual differences among attachment styles in the subliminal priming paradigm (Pierce \& Lydon, 1998; Banse, 1999, 2001; Mikulincer et al. 2001, Study 1-5; Mikulincer \& Shaver, 2001). Based on these findings, our second hypothesis is that there are no significant differences among attachment avoidance and anxiety in the affective priming paradigm.

In terms of culture differences, it is difficult to make a hypothesis in advance. To our knowledge, there are few studies on adult attachment using a Chinese sample. Man \& Hamid (1998) surveyed college students in Hong Kong and found similar proportions of secure, dismissing, preoccupied, and fearful to western samples. Our data have also showed more secure type in mainland of China ( $\mathrm{Li} \&$ Kato, in press), and more preoccupied type in Japan (Nakao \& Kato, 2004). However, because there is an unique association between secure base and amae in Japanese subjects (Rothbaum et al., 2000), it is possible that Chinese subjects rather than Japanese subjects will get similar results to previous studies (e.g., Mikulincer, et al., 2001).

\section{Study 1: Using Affect Priming in a Japanese Sample}

In this study, we examined the associative processing mode of secure base schema in Japanese sample. Participants were presented with 40 neutrally valued Nepalese characters and asked to rate their liking for each target. In each trial, immediately before the target, participants were presented by one of four prime pictures. 
We wanted to know whether secure base schema results in positive affect in participants, and then make them misattribute the positive affect to neutral target stimuli, and whether the priming effect of secure base schema depends on one's personal attachment style. To measure attachment style, participants are asked to complete Brennan et al.'s (1998) ECR scale, adapted by Nakao \& Kato (2004).

\section{Method}

\section{Participants}

Fifty five undergraduate and graduate students from Kyushu University (34 men and 21 women ranging from 20 to 36 years old, median=23) volunteered to participate in the study without any monetary reward. All had normal or corrected-to-normal vision.

\section{Materials}

We used four prime pictures: two secure base prime pictures (a black-and-white Picasso's sketch and a Japanese picture), one positive prime picture (a happy face) and one neutral prime picture (a random black-and-white polygon). Picasso's sketch was a 1922 pen-and-ink realistic drawing, depicting a mother (western female face) holding and looking at her baby. A graduate student who is well trained in drawing drew the Japanese picture, depicting an eastern mother holding and looking at her baby. The happy face picture was a smiling face of a young eastern female without visible clothing who was photographed against a gray background.

Although both Murphy \& Zajonc (1993) and Mikulincer et al. (2001) use Chinese ideographs as target stimuli, they cannot test on Japanese and Chinese sample, because both Chinese and Japanese subjects may know the ideographs well. We decided to use Nepalese characters as our target stimuli. We chose 65 Nepalese characters from an introduction book based on their physical characteristics (i.e., line, circle, space, length, width, etc.).

We conducted a pretest to validate the categorization of the 4 prime pictures and the neutrality, novelty and ambiguity of the target stimuli. Twenty undergraduate or graduate students ( 8 female, 12 male) volunteered for the pretest. All had normal or 
corrected-to-normal vision. All participants evaluated the prime pictures, while 15 out of 20 participants evaluated the target stimuli. We sequentially presented participants with the four prime pictures on the computer screen. For each picture, subjects were asked to rate the extent to which the picture (1) reflects themes related to interpersonal relationships, (2) arouses feelings of warmth and love, (3) arouses positive affect; and (4) is familiar with. These ratings were performed on a 7-point scale ranging from not at all (1) to very much (7).

The results of evaluation on prime pictures are as below: For themes related to interpersonal relationships, ANOVA analysis yields a significant main effect for prime pictures on 7-point rating, $\mathrm{F}(3,57)=79.33, \mathrm{p}<.01$. Japanese picture $($ Mean $=5.70)$ has the highest rating, then Picasso picture (Mean $=5.10)$, followed by Happy face (Mean = 2.25) and Polygon has the lowest rating (Mean=1.40). For aroused feelings of warmth and love, participants rated Japanese picture $($ Mean $=6.35)$ arousing more warm and love feeling than Picasso's sketch (Mean = 5.55), Happy face $($ Mean $=2.98)$, and Neutral polygon $($ Mean $=1.35), F(3,57)=106.0, p<.01$. In addition, participants rated Japanese picture arousing more positive affect $($ Mean $=5.55)$ than Picasso's sketch $($ Mean $=5.05)$ and Happy face $($ Mean $=4.55)$, neutral polygon led to lowest rating $($ Mean $=2.35), F(3,57)=19.36, \underline{p}<.01$. Finally, participants reported that they are more familiar with Japanese picture (Mean=5.40) than Picasso's sketch (Mean=4.80) and Happy face (Mean=4.15), Polygon led to lowest rating (Mean=2.45), F $(3,57)=$ $28.35, \mathrm{p}<.001$. These findings validate our prime picture categorization.

To validate the neutrality, novelty, and ambiguity of the target stimuli, we asked subjects to rate on computer (1) the familiarity with each of the 65 stimuli, (2) the extent to which each stimulus arouses positive affect, (3) the extent to which each stimulus arouses negative affect, (4) the extent to which they could ascribe a meaning to each of the stimuli. The ratings were made on 7-point scale range from not at all (1) to very much (7). In this procedure, each target picture was presented on the upper part of the screen until all 4 questions were answered. Questions were serially presented at the center, and 7-point scale on the bottom of screen. After subject rated all 4 questions, next target picture was presented. We considered that neutrality is associated with low familiarity, low positive affect arousal, low negative affect arousal and meaningless for subjects, so that the average score of four questions is used as an index of neutrality. Based on this index, we chose the lowest 40 as our target pictures, and 5 for practice pictures. A total of 15 undergraduate or graduate students ( 7 female, 8 male) 
volunteered for this program. The 40 target pictures were rated as unfamiliar stimuli (Mean= 2.23, Range=1.80-2.53). Moreover, they were rated as being affectively neutral (Positive affect arousing: Range=1.73-2.33, Mean=1.92; Negative affect arousing: Range=1.87-2.73, Mean= 2.31) and meaningless (attached meaning: Range=1.20-1.67, Mean $=1.36$ ). We also ask subjects to rate the extent to which they like each of the target stimuli in the condition without prime. Each target was presented $2000 \mathrm{~ms}$, followed by a 7-point scale. After subject's response, next target was presented. This program is similar to the experiment (see method in Study 1) except that there is no prime stimulus. The mean liking rating for these 40 target pictures with conscious awareness was 3.49 , ranging from 2.93 to 3.80 . These findings validated the choice of the 40 target pictures.

In the experiment, the prime pictures are 4 drawings, which were validated in the pretest, and another non picture prime condition. The target pictures are 40 Nepalese characters validated in pretest. Based on the pretest, we composed 40 trials by putting together a specific prime picture and a specific Nepalese character. Each prime picture was presented in 8 trials. The prime-target combinations and the order of presentation of these combinations were randomized across participants. Each participant received different, random combinations of prime pictures and target pictures that were presented in a different, random order.

\section{Procedure}

Participants were individually invited to laboratory, seated in front of a computer that provided all the instructions, and told to complete a computerized "visual perception" task. The task consisted of 40 trials, run on a SONY Pentium III computer with an SVGA color monitor. Brightness and contrast were set low, and the primes and target pictures, each with the size of $6.0 \mathrm{~cm}$ width and $6.8 \mathrm{~cm}$ height, were displayed in black on a gray background in the middle of the screen. At a viewing distance of $80 \mathrm{~cm}$, the dimensions of the picture stimuli were $4.30^{\circ}$ (width) and $4.87^{\circ}$ (height) of visual angle. The experiment program was written by the authors in Visual Basic 6.0.

The sequence of the events on each trial is schematized in Fig. 1. On each trial, a fixation point (a black X) was displayed in the center of the screen for $1000 \mathrm{~ms}$. At the fixation point offset, a randomly chosen prime picture was exposed for $10 \mathrm{~ms}$, followed by a rain pattern of gray dots for $500 \mathrm{~ms}$, which served as a backward mask. Then a 
randomly chosen Nepalese character was presented for 2,000ms. At the offset of the Nepalese character, a 7-point Likert scale, ranging from not at all (1) to very much (7), was presented on the screen, subjects were told to rate the extent to which they liked the character, using the Mouse to choose a number. When they made a rating, the scale vanished and, after a 2 s pause, the next trial began.

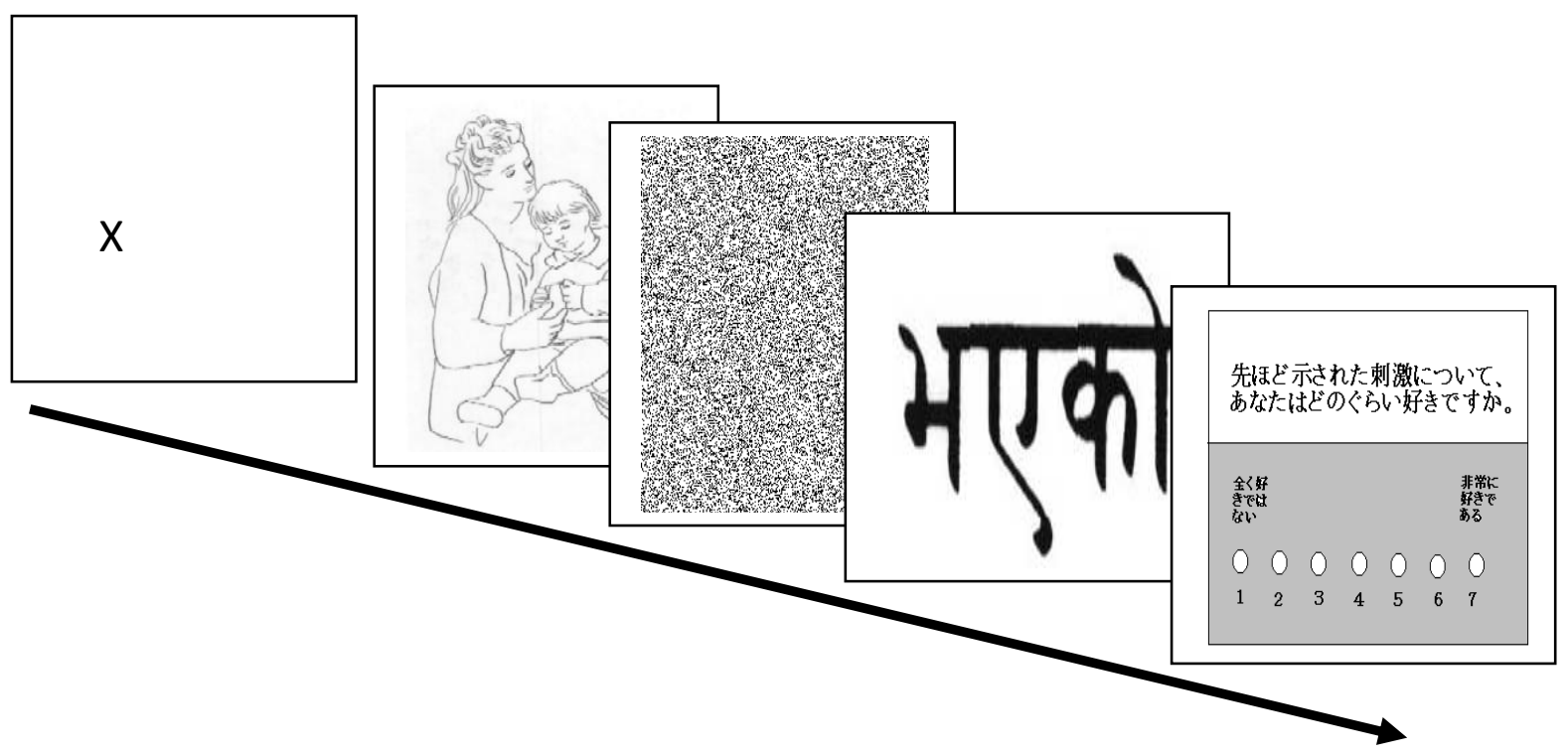

$1000 \mathrm{~ms}$

$10 \mathrm{~ms}$

$500 \mathrm{~ms}$

$2000 \mathrm{~ms}$

Liking rating

Figure 1

Sequence of the events on each trial of Study 1 
Participants began with 5 practice trials. In the practice trials, there was no prime picture, and another 5 Nepalese characters served as practice target stimuli. After 5 practices, they had completed 40 trials. When they finished this "visual perceptual task", they filled in the computer-based questionnaire on Adult Attachment Scale (36item scale, originally developed by Brennan et al., 1998, Japanese version was adapted by Nakao \& Kato, 2004). This scale measured the attachment dimensions of anxiety and avoidance with 18 -items on attachment anxiety (e.g., "I worry about being abandoned", "I find that other persons don't want to get as close as I would like") and another 18-items on attachment avoidance (e.g., "I try to avoid getting too close to other persons", "I get uncomfortable when other persons want to be very close). Participants rated the extent to which an item described their feelings in close relationships, using a 7-point scale ranging from not at all (1) to very much (7). Participants were asked to answer attachment style scale only after completing the evaluation task to avoid any effect of the activation of chronic attachment related schemas during the completion of attachment scale on participant's evaluations of the target stimuli.

Before ending the experiment, we asked participants whether they saw more than a Nepalese character flashed on the screen. All participants reported that they have seen only one Nepalese character in each display, that is, none of the participants was able to detect the subliminal primes or to discern anything about the valence or meaning of these prime pictures. In the end, participants were debriefed on the real purpose of the experiment and thanked for their participation.

\section{Results and Discussion}

We averaged each participant's liking ratings of the targets in each of the priming categories. The results were showed in Table 1. Then we conducted a one-way repeated measurement ANOVA for prime pictures (Japanese/Indigenous picture, Picasso, Happy face, Polygon, Non-picture) on the averaged liking ratings. The ANOVA yielded no significant differences among 5 prime pictures, $F(4,276)=0.73$, $\mathrm{p}=0.57$, with similar ratings for Japanese picture (Mean $=3.61$ ), Picasso's sketch $($ Mean $=3.58)$, Happy face $($ Mean $=3.58)$, Polygon $($ Mean = 3.67) and Non-picture $($ Mean $=3.66)$. 
Table 1. Means and Standard Deviations of Liking Ratings by Prime Pictures

\begin{tabular}{cccccc}
\hline & Indigenous Picture $^{1}$ & Picasso's Sketch $^{\text {Happy Face }}$ & Polygon & No picture \\
\hline \multicolumn{2}{l}{ Study 1: Japanese sample, $\underline{\mathrm{n}}=55$} & & & & \\
\hline Mean & $3.61_{\mathrm{c}}$ & $3.58_{\mathrm{c}}$ & $3.58_{\mathrm{c}}$ & $3.67_{\mathrm{c}}$ & $3.66_{\mathrm{c}}$ \\
SD & 0.90 & 0.79 & 0.85 & 0.94 & 0.79 \\
\hline \multicolumn{2}{l}{ Study 2: Chinese sample, $\underline{\mathrm{n}}=60$} & & & & \\
\hline Mean & $4.17_{\mathrm{a}}$ & $4.00_{\mathrm{ab}}$ & $3.91_{\mathrm{b}}$ & $3.87_{\mathrm{b}}$ & $3.71_{\mathrm{c}}$ \\
SD & 0.88 & 0.85 & 0.96 & 0.98 & 0.80 \\
\hline
\end{tabular}

Note: ${ }^{1}$ Indigenous picture means Japanese picture for Japanese subjects, Chinese picture for Chinese subjects. Means with different subscripts are significantly different at a two-sided significance level of 0.05 .

In examining the moderating effects of a participant's attachment style, the 36 items of the attachment style were factor analyzed. A principal components method with varimax rotation for orthogonal factors was conducted in order to test for the twofactor structure proposed by Brennan et al. (1998). Two factors were extracted for the final solution, accounting for $40 \%$ of the variance. The item content of the two factors closely matched the original scale. According to Brennan et al. (1998), of the 36 items, 18 items were clustered to form anxiety factor (Cronbach's $\alpha=0.87$ ) and their average is adopted as an index of anxiety. The other 18 items were grouped under the avoidance factor (Cronbach's $\alpha=0.91$ ) and their average is adopted as an index of avoidance. Pearson's correlation yielded a modest association between the two attachment scores, $\underline{r}$ $=0.21$, emphasizing an orthogonality of the two attachment dimensions. Mean of anxiety and avoidance are $3.28(\mathrm{SD}=1.08)$, and $3.58(\mathrm{SD}=0.90)$ respectively. In addition, there is no significant gender effect on each of the two attachment scores (For anxiety, $F(1,53)=0.02, \underline{p}=0.89$, for avoidance, $F(1,53)=1.89, \underline{p}=0.18)$.

We conducted a three-way ANOVA on the liking rating, with attachment anxiety (below median, above median), attachment avoidance (below median, above median), and prime pictures (Picasso's sketch, Japanese picture, Happy face, Polygon, Non picture). The last factor was a within-subject repeated measure. This analysis yielded no significant main effect for prime picture $(F(4,204)=.70, \underline{p}=.59)$ or for anxiety $(F$ $(1,51)=.20, \underline{p}=.66)$ or for avoidance $(F(1,51)=4.54, \underline{p}=.06)$. In addition, there was no significant two-way interactions (for Prime X Anxiety, F $(4,204)=1.82, \underline{p}=.13$; for Prime $X$ Avoidance, $F(4,204)=1.20, p=.31)$ or three-way interaction $(F(4,204)=$ $1.63, \mathrm{p}=.17)$.

Overall, Mikulincer et al.'s (2001) results cannot be replicated in Japanese sample. Secure base schema did not led to higher rating on neutral stimuli. The mean ratings 
were at the same level for positive prime, neutral prime and no prime. Such findings also indicated that attachment style had no effects on the priming in Japanese sample.

\section{Study 2: Using Affect Priming in a Chinese Sample}

Either Picasso's sketch or Japanese picture is art composition, not a symbol in the real-life. In order to find an attachment symbol in the real life, i.e., a most familiar picture of secure base, we conducted a similar study in Lanzhou, a big city in the northwest China located at the upper reaches of Yellow river. Breeding one of the origins of Chinese culture, Yellow rive is considered the Mother river of China and the people in Lanzhou take a great pride in its famous city symbol, a sculpture known as "Yellow River's Mother" (Huang He Mu Qin). The landmark sculpture features a son climbing on mother's body, and mother looking at her son. Like Picasso's sketch, it depicts the core component of secure base schema: receipt a caring and comfort with primary attachment figure.

\section{Method}

\section{Participants}

Sixty undergraduate and graduate students (35 women and 25 men, ranging from 19 to 34 years old, median=22) from Northwest China Normal University, Lanzhou, P. R .China, volunteered to participate in the study without any monetary reward. All had normal or corrected-to-normal vision.

\section{Materials and Procedure}

The prime pictures were similar to Study 1, except using the Chinese picture "Huang He Mu Qin" instead of the Japanese picture. The target stimuli were 40 Nepalese characters, the same as those in Study 1.

The same experiment program was used in Chinese sample with the only exception that after completing task, participants filled out a Chinese version of Brennan's attachment scale (Li \& Kato, 2006). 
To validate the prime picture, we also conducted a pretest with other 47 students in Chinese sample. Using the same procedure as pretest in Japanese sample, we presented participants with the Picasso's picture, Chinese picture, Happy face, Polygon and Nonpicture serially on the computer screen. The results of evaluation on prime pictures as below: For themes related to interpersonal relationships, ANOVA analysis yielded a significant main effect of prime effect on ratings, $F(3,138)=111.59, \mathrm{p}<.001$.. Chinese picture $($ Mean $=5.38)$ had the highest rating, then Picasso picture (Mean $=$ 4.72), followed by Happy face (Mean $=2.94$ ) and Polygon had the lowest rating (Mean $=2.17$ ). For aroused feelings of warmth and love, MANOVA revealed that participants rated Chinese picture $($ Mean $=5.36)$ arousing more feelings of warmth and love than Picasso's sketch $($ Mean $=4.74)$, Happy face $($ Mean $=3.55)$, and Neutral polygon (Mean $=2.47), \mathrm{F}(3,138)=86.12, \mathrm{p}<.01$. In addition, participants rated Chinese picture arousing more positive affect $($ Mean $=5.32)$ than Picasso's sketch $($ Mean $=4.77)$ and Happy face $($ Mean $=4.72)$, neutral polygon led to lowest rating (Mean = 3.28), F (3, $138)=29.51, \mathrm{p}<.01$. Finally, participants reported that they are more familiar with Chinese picture (Mean=6.19) than Picasso's sketch (Mean=4.40) and Happy face (Mean=4.26), Polygon led to lowest rating $($ Mean=2.85), F $(3,138)=124.10, \mathrm{p}<.001$. These findings validated our prime picture categorization in Chinese sample.

\section{Results and Discussion}

We averaged each participant's liking ratings of the targets in each of the priming categories. The results were also showed in Table 1. We conducted a one-way repeated measurement ANOVA for prime pictures (Chinese picture, Picasso, Happy face, Polygon, Non picture) on the averaged liking ratings. The ANOVA found significant

main effect for prime pictures, $F(4,236)=3.93, \underline{p}<.01 . \eta^{2}=.06$. Further tests for repeated measures revealed that the secure base prime pictures (Picasso's sketch: Mean $=4.00$, Chinese Picture: Mean=4.17) had higher liking ratings of target stimuli than did Happy face $($ Mean $=3.91)$ or Polygon $($ Mean $=3.87)$ or no picture $($ Mean $=3.71)$.

In examining the moderating effects of a participant's attachment style, the 36 items of the Chinese version of attachment style were factor analyzed using the same procedure for Japanese sample. Two factors were extracted for the final solution, accounting for $37 \%$ of the variance. The item content of the two factors closely matched the original scale. 18 items were clustered to form anxiety factor (Cronbach's $\alpha=0.69$ ), 
so we averaged these items as an index of anxiety. The other 18 items were grouped under the avoidance factor (Cronbach's $\alpha=0.84$ ), we also averaged these items as an index of avoidance. Pearson's correlations yielded a modest association between the two attachment scores, $\underline{r}=0.15$, indicate an orthogonality of the two attachment dimensions. Mean of anxiety and avoidance are $3.55(\mathrm{SD}=0.85)$, and $4.18(\mathrm{SD}=0.68)$ respectively. In addition, there is no significant gender effect on each of the two attachment scores (for anxiety, $\mathrm{F}(1,58)=0.06, \mathrm{p}=.81$, for avoidance, $\mathrm{F}(1,58)=0.19$, $\mathrm{p}=.67)$.

Similar three-way ANOVA on the liking rating, with attachment anxiety (below median, above median), attachment avoidance (below median, above median), and prime pictures (Picasso's sketch, Chinese picture, Happy face, polygon, no picture) shows significant main effect of prime pictures $(F(4,224)=3.99, p<.01)$, but not for the main effect of anxiety $(F(1,56)=0.11, p=.74)$ or avoidance $(F(1,56)=0.24, p=$ .62). In addition, there was no significant two-way interactions (for Prime X Anxiety, F $(4,224)=1.70, p=.15$; for Prime $X$ Avoidance, $F(4,224)=.77, p=.55)$ or three-way interaction $(\mathrm{F}(4,224)=1.04, \underline{p}=.39)$.

\section{Comparison of the Japanese with the Chinese sample}

In order to compare the two samples, we conducted a two-way MANOVA for country (Japanese, Chinese) and prime picture (Picasso's sketch, Indigenous picture, Happy face, Polygon, Non picture) on the averaged liking ratings. The later factor was a within-subject measure. This MANOVA revealed a significant main effect for country, $\mathrm{F}(1,113)=5.16, \mathrm{p}<.05$, and prime pictures, $\mathrm{F}(4,452)=2.16, \underline{\mathrm{p}}<.01$, and a significant interaction, $\mathrm{F}(4,452)=3.69, \mathrm{p}<.01$. Overall, Chinese subjects (Mean $=$ $3.93, \mathrm{SD}=0.67)$ rated target stimuli higher than Japanese subjects (Mean $=3.62, \mathrm{SD}=$ 0.78), however, this effect was qualified by a significant interaction. They rated much higher for Indigenous picture (Chinese picture) than their Japanese counterparts for Japanese picture, $\mathrm{F}(1,113)=7.49, \mathrm{p}<.01$ (add the estimated mean ratings for indigenous pictures). They also rated higher for Picasso's sketch and Happy face than Japanese subjects $(\mathrm{F}(1,113)=11.01, \mathrm{p}<.01, \mathrm{~F}(1,113)=3.90, \mathrm{p}<.05$, respectively $)$. But for polygon and no picture, there were no significant differences between the two samples $(\mathrm{F}(1,113)=1.15 ; \mathrm{F}(1,113)=0.09$, all $\underline{\mathrm{p}}>.05)$. In addition, there were no significant differences on attachment anxiety $(\mathrm{F}(1,113)=2.09, \mathrm{p}=0.152)$, while 
significant differences on attachment avoidance $(\mathrm{F}(1,113)=16.32, \mathrm{p}<.01)$ between Japanese and Chinese participants.

These findings partly support Mikulincer et al. (2001) results in Chinese sample. The subliminal priming of a secure base representation led to more positive evaluation of neutral stimuli than did the subliminal priming of neutral or no pictures. The most familiar secure base picture ("Huang He Mu Qin") led to the highest liking rating on neutral target. In terms of culture difference, the positive affect can be activated by secure base picture in Chinese sample but not in Japanese sample, while there are no differences between the two samples in polygon and no prime pictures conditions.

\section{General Discussion}

The current research reveals some interesting findings on the affective component of the secure base schema. First, it suggests that a cultural dimension may be added to Mikulincer et al. (2001)'s argument on the affective component of secure base schema. Second, it provides a new insight on the associative processing mode of affect component in the secure base schema. Third, they yield new evidence that attachment style has no effect on the evaluation of neutral target stimuli in subliminal priming paradigm.

"Amaenbou", a Japanese word, means a spoiled, pampered child or a child who makes up to people. Those "amaenbou" are always expected to be loved (Kato, 1994, 1995) and to depend on others (Markus \& Kitayama, 1991). Interestingly, there is another Chinese word "Xiao Huang Di", with a literal meaning of "little emperor", for Chinese willful, capricious only-children generation (Falbo \& Poston, 1993; Jiao, Ji \& Jing, 1996). Those "Xiao Huang Di" are always expected to have high achievements. The two words may exactly reflect the basic differences in early childrearing between Japanese and Chinese. Is there any difference between "amaenbou" and "Xiao Huang Di" when they grow up? Our studies explored their differences on secure base of adult attachment using subliminal affect priming experiments.

In the reported studies, subliminally priming of secure base representations led to more positive affective reactions to neutral stimuli only in the Chinese sample. In the Japanese sample, there are no significant differences among four prime pictures. Given that in the pretest (with conscious awareness) both Japanese and Chinese made similar scores on liking-rating task for all prime pictures, the differences on the subliminal 
affective priming effect of secure base pictures may reflect the differences of associations in the secure base schema. This subliminal priming effect may be different from the affect expression with conscious awareness.

According to the dual-process model in social psychology (Smith \& DeCoster, 2000), the associative processing performs pattern completion function. When similar cues were presented, the associative process uses currently available cues to retrieve representations of past occasions. In affective priming paradigm, the prime pictures can automatically activate the related node of a semantic network (Fazio, 2001; GinerSorolla, Garcia \& Bargh, 1999; Spruyt, Hermans, De Houwer, \& Eelen, 2002). Once secure base pictures were presented, the associations between self and attachment figure would be activated automatically. But the activated associations between Japanese and Chinese may be different.

In our Japanese sample, the liking ratings were essentially at the same level for all prime pictures. At the first glance, these results are at odds with Mikulincer et al. (2001) in that secure base pictures did not lead to positive affect. But it is consistent with previous studies using Japanese participants (e.g., Ogawa \& Suzuki, 1998), which failed to find priming effects using the affective priming paradigm. For Japanese participants, their associations with attachment figure may focus on dependence. Evidence of this dependence is that Japanese children tend to stay with their mothers, seldom separate from their mothers (Takahashi, 1990), and even in their adulthood, they still depend on their parents or spouse as an adaptive attachment strategy (Onishi \& Gjerde, 2002). On the other hand, as a result of "cultural expectation", to strive to remove any kind of stress for children is the main work of the mothers in Japanese society. When their children express wishes to be independent in adolescence, many mothers feel a loss of purpose and experience an empty-nest syndromes (Kazui, 1997). It is reasonable that those secure base pictures may also reflect other aspects of interpersonal relationship, such as dependence, in our Japanese sample.

On the contrary, secure base pictures did lead to higher positive affect in Chinese sample. For Chinese participants, the associations between themselves and attachment figures may focus on their academic achievements. In China, childrearing is the main work of all members of the 'big family' especially after the "only-children" policy was officially implemented in 1980s. This policy has radically changed the Chinese family structure, raised great concern in the society about the change in parenting style (Yang, Ollendick, Dong, Xia, and Lin, 1995; Wang, and Ollendick, 
2001). Big family rather nuclear family is usual in China, because of filial piety cultural tradition and housing shortages for the younger generations. Each infant is often the only child in the big family living with the grandparents from both sides of parents' family. Such a configuration was thought to spoil the child, leading them to behave like 'little emperors' (Falbo \& Poston, 1993). On the other hand, Chinese families have traditionally been 'authoritarian', which is represented by endorsement of frequent use of power assertive, prohibitive, and punitive strategies (Chen, Liu, Li, Cen, Chen and Wang, 2000), and high expectation of academic achievement (Falbo \& Poston, 1993; Jiao, Ji \& Jing, 1996), high parental involvement (Wang \& Ollendick, 2001). Thus, the only-child in the family is overprotected, carrying heavy burden to make high achievement at the same time. For these Chinese youth, secure base pictures activated achievement related representations of attachment figure. Their primed affection is likely more positive than that of their Japanese counterparts.

Yet, we should note that Chinese picture causes the highest liking rating, Picasso's sketch and positive picture lead to the same ratings as neutral polygon prime in Chinese sample. This may indicate an effect of familiarity of the prime. The more familiar the prime, the stronger activation of the related association it leads to (Ye \& van Raaij, 1997). But the role of familiarity may be mediated by the nature of associations. For Japanese sample, activated amae-related association is more neutral and therefore even more familiar picture (Japan picture) can not lead to a higher liking rating.

Another important question concerns the effect of attachment styles on the primed attachment affect. In both Japanese and Chinese sample, it failed to find any differences among attachment styles. It may imply that there are no meaningful individual differences at the subliminal priming level (Banse, 1999). Thus, automatically primed attachment affection as assessed in the affective priming paradigm is structurally independent from the processes such as evaluation of relationship satisfaction in the sense of Brennan et al. (1998). Affective priming may reflect a quick but general evaluation that is directly linked to the associative processing mode of attachment figure (e.g., Fazio et al., 1986). However, it is not to mean that primed attachment affection is independent of attachment style, because those secure base pictures may not be the adaptive situation to trigger the attachment system. In fact, there are effects of attachment styles on liking ratings in stress situations (Mikulincer et al., 2001). Moreover, secure base is not the only function of attachment. There are some other functions, such as safe haven (Shaver \& Mikulincer, 2002). It is possible that if we use 
some prime pictures that can represent safe haven function of attachment, effect of attachment style may be found. Further study in this direction is warranted.

It is important to point out that our studies are the first step to examine the affective component of secure base schema in the dual-process model domain. There are limitations in the current studies. First, the associative processing mode in secure base was assessed by making liking rating for neutral target stimuli under different prime pictures ---- this is only an indirect measurement. Maybe it is better to use some other priming paradigm such as evaluation task (e.g., Fazio et al., 1986), which asks participants to make judgment for positive or negative target stimuli to assess the association between secure base and positive affect. Second, in our experiments each prime picture was presented 8 times, thus one could argue that there is a mere exposure effect on subliminal priming (Monahan, Murphy, and Zajonc, 2000; Zajonc, 2001). Moreover, different indigenous pictures were used for Japanese and Chinese subjects in the current studies. Given the similarity of physical feature of Asian pictures, using Japanese picture to test on Chinese subjects is needed. Finally, our research's purpose is exploratory, and no culture contextual variables are included. It is just a psychological-differences study, further research would extend to generalizability studies (van de Vijer \& Leung, 1997, 2001), focus on the evaluation of a hypothesized cultural difference or similarity in the absence of contextual variables. These limitations suggest that it is necessary to replicate our findings with other subliminal priming paradigm.

\section{References}

Ainsworth, M.D.S. (1989). Attachments beyond infancy. American Psychologist, 44,709-716.

Ainsworth, M.D.S., Blehar, M.C., Waters, E., \& Wall, S. (1978). Patterns of attachment: A psychological study of strange situation. Hillsdale, NJ: Erlbaum.

Baldwin, M.W., Keelan,J.P.R., Bever;eu Fehr, Vicki Enns, \& Evelyn Koh-Rangarajoo. (1996). Social-Cognitive Conceptualization of Attachment Working Models: Availability and Accessibility Effects. Journal of Personality and Social Psychology, 71 94-109.

Banse, R. (1999). Automatic evaluation of self and significant others: affective priming in close relationships. Journal of social and personal relationships, 16, 803-821. 
Banse, R. (2001). Affective priming with liked and disliked persons: prime visibility determines congruency and in-congruency effects. Cognition and emotion. 15, 501-520.

Bowlby, J. (1969/1997). Attachment and Loss: Attachment. Pimlico.

Bowlby, J. (1973/1998). Attachment and Loss: Separation. Pimlico.

Bowlby, J. (1980/1998). Attachment and Loss: Loss. Pimlico.

Brennan, K.A., Clark, C.L., \& Shaver, P.R. (1998). Self-report measurement of adult attachment: an integrative overview. In J.A. Simpson \& W. S. Rholes (Eds.), Attachment theory and close relationships. New York: The Guilford Press. Pp. 4676.

Carver, C.S. (2001). Affect and the functional bases of behavior: On the dimensional structure of affective experience. Personality and Social Psychology Review, 5, 345-356.

Chen, x., Liu, M., Li, B., Cen, G., Chen, H. \& Wang, L. (2000). Maternal authoritative and authoritarian attitudes and mother-child interactions and relationships in urban China. International Journal of Behavioral Development, 24, 119-126.

Colins,V.L.(1996). Human Attachment. Temple University Press.

Crittenden, P. M. (2000). A dynamic-Maturational Exploration of the Meaning of Security and Adaptation: Empirical, Cultural, and Theoretical Consideration. In Crittenden, P.C., \& Claussen, A.H. (Eds.), The Organization of Attachment Relationships: Maturation, Culture, and Context. (Chapter 19, pp. 358-383). Cambridge University Press.

Davis, J.T. (2001). Gone but not forgotten: Declarative and nondeclarative memory processes and their contributions to resilience. Bulletin of the Menninger Clinic, $65,451-470$.

Falbo, T. \& Poston, D.L. Jr. (1993). The academic, personality, and physical outcomes of only children in China. Child Development, 64, 18-35.

Fazio,R.H. (2001). On the automatic activation of associated evaluations: An overview. Cognition and Emotion. 15, 115-141.

Fazio, R.H., Sanbonmatsu, D.M, Powell, M.C. \& Kardes, F.R. (1986). On the automatic activation of attitudes. Journal of Personality and Social Psychology, 50, 229-238.

Fraley, R.C., \& Shaver, P.R. (1998). Airport Separations: A Naturalistic Study of Adult Attachment Dynamics in Separating Couples. Journal of Personality and Social 
Psychology, 75, 1198-1212.

Fraley, R.C., Garner, J.P., \& Shaver, P.R. (2000). Adult attachment and the defensive regulation of attention and memory: Examining the role of preemptive and postemptive defensive processes. Journal of Personality and Social Psychology, $79,816-826$.

Fuendeling, James.M. (1998). Affect regulations as a stylistic process within adult attachment. Journal of Social \& Personal Relationships. 15, 291-322.

Giner-Sorolla, R., Garcia, M.T., \& Bargh, J.A. (1999). The automatic evaluation of pictures. Social Cognition, 17, 76-96.

Hazan, C., \& Shaver, P. (1987). Conceptualizing romantic love as an attachment process. Journal of Personality and Social Psychology, 52, 511-524.

Jiao, S., Ji, G. \& Jing, Q. (1996). Cognitive development of Chinese urban only children and children with siblings. Child development, 67, 387-395.

Kato, K. (1994/5). An Analysis of Amae Processes and Interactions: A Review and A Proposal of Process Models. Journal of Cognitive Process and Experiencing. 4, 126.

Kazui, M. (1997). The influence of Cultural Expectations on Mother-child Relationship in Japan. Journal of Applied Developmental Psychology, 18, 485-496.

Knox, J. (1999). The relevance of attachment theory to a contemporary Jungian view of the internal world: internal working models, implicit memory and internal objects. Journal of Analytical Psychology. 44, 511-530.

Knox, J. (2001). Memories, fantasies, archetypes: an exploration of some connections between cognitive science and analytical psychology. Journal of Analytical Psychology, 46, 613-635.

Knox, J. (2003). Trauma and defenses: their roots in relationship. Journal of Analytical psychology, 48, 207-233.

Li, T.G., \& Kato, K. (2006). Measuring adult attachment: Chinese validation of ECR. Acta Psychologica Sinica, 38, 399-406.

Lyddon, W.J., Bradford, E., \& Nelson, J.P. (1993). Assessing adolescent and adult attachment: A review of current self-report measures. Journal of Counseling \& Development. 71, 390-395.

Lyons-Ruth, K. (1998). Implicit relational knowing: Its role in development and psychoanalytic treatment. Infant Mental Health Journal, 19, 282-289.

Main, M., Kaplan, N., \& Cassidy, J. (1985). Security in infancy, childhood, and 
adulthood: A move to the level of representation. In I. Bretherton \& E. Waters (Eds.), Growing points in attachment theory and research. Monographs of the Society for Research in Child Development, 50, 66-104.

Man,K.O., \& Hamid,P.N. (1998). The relationship between attachment prototypes, selfesteem, loneliness and causal attributions in Chinese trainee teachers. Personality \& Individual Differences. 24,357-371.

Markus, H. \& Kitayama, S. (1991). Culture and the self: Implications for cognition, emotion, and motivation. Psychological Review, 98, 224-253.

Mayer, B. \& Merckelbach, H. (1999). Unconscious processes, subliminal stimulation, and anxiety. Clinical Psychology Review, 19, 571-590.

Mikulincer, M., Girschberger, G., Nachmias, O., \& Gillath, O. (2001). The Affective Component of the Secure Base Schema: Affective Priming With Representations of Attachment Security. Journal of Personality and Social Psychology, 81, 305-321.

Mikulincer, M., Shaver, P. R. (2001). Attachment Theory and Intergroup Bias: Evidence That Priming the Secure Base Schema Attenuates Negative Reactions to Out-groups. Journal of Personality and Social Psychology, 81, 97-115.

Mizuta, I., Zahn-Waxler, C., Cole, P.M. \& Hiruma, N. (1996). A cross-cultural study of preschoolers' attachment: Security and sensitivity in Japanese and US dyads. International Journal of Behavioral Development, 19, 141-159.

Monahan, J.F., Murphy, S.T. \& Zajonc, R.B. (2000). Subliminal mere exposure: Specific, general, and diffuse effects. Psychological Science. 11, 462-466.

Murphy, S.T., \& Zajonc, R.B. (1993). Affect, cognition, and awareness: Affective priming with optimal and suboptimal stimulus exposures. Journal of Personality and Social Psychology, 64, 723-739.

Murphy, S.T., Monahan, J.L. \& Zajonc, R.B. (1995). Additivity of nonconscious affect: Combined effects of priming and exposure. Journal of Personality and Social Psychology, 69, 589-602.

Nakao, T. \& Kato, K. (2004). Seijin aichaku style shakudo (ECR) no nihongoban sakusei no kokoromi. Shirigaku Kenkyu. [Constructing the Japanese version of the adult attachment style scale. The Japanese Journal of Psychology, 75 (2):154-159.

Ogawa, T. \& Suzuki, N. (1998). Ikika kanjyoteki Priming kouka no kentou. Kanjyoshirigaku kenkyu. [An examination of the subliminal affective priming effect]. The Japanese Journal of Research on Emotions, 5, 70-77.

Onishi, M., \& Gjerde, P.F. (2002). Attachment strategies in Japanese urban middle-class 
couples: A cultural theme analysis of asymmetry in marital relationships. Personal Relationships, 9, 435-455.

Pierce, T., \& Lydon, J. (1998). Priming relational schemas: Effects of contextually activated and chronically accessible interpersonal expectations on responses to a stressful event. Journal of Personality and Social Psychology, 75, 1441-1448.

Pietromonaco, P.R.\& Barrett Feldman, L. (1997). Working models of attachment and daily social interactions. Journal of Personality \& Social Psychology, 73, 14091423.

Pietromorcuo, P., Barrett,Lisa,F. (2000). The internal working models concept: what do we really know about the self in relation to others? Review of General Psychology. 4, 155-175.

Rothbaum, F., Pott, M., Azuma, H., Miyake, K. \& Weisz, J. (2000). The development of close relationships in Japan and the United States: Paths of Symbiotic Harmony and Generative Tension. Child Development, 71, 1121-1142.

Rothbaum, F., Rosen, K., Ujiie, T., \& Uchida, N. (2002). Family systems theory, attachment theory, and culture. Family Process, 41, 328-350.

Rothbaum, F., Weisz, J., Pott, M., Miyake, K., \& Morelli, G. (2000). Attachment and culture: Security in the United States and Japan. American Psychologist, 55, 10931104.

Rowe, A. \& Carnelley, K.B. (2003). Attachment style differences in the processing of attachment-relevant information: Primed-style effects on recall, interpersonal expectations, and affect. Personal Relationships, 10, 59-75.

Shaver, P. R. \& Mikulincer, M. (2002). Dialogue on adult attachment: Diversity and integration. Attachment \& Human Development, 4, 243-257.

Smith, Eliot R; De Coster, Jamie. (2000). Dual-process models in social and cognitive psychology: Conceptual integration and links to underlying memory systems. Personality \& Social Psychology Review. 4, 108-131.

Spruyt, A., Hermans, D., de Houwer, J. \& Eelen, P. (2002). On the nature of the affective priming effect: affective priming of naming responses. Social Cognition, 20, 227-256.

Takahashi, K. (1990). Are the Key Assumptions of the "Strange Situation" Procedure Universal? A View from Japanese Research. Human Development, 33, 23-30.

Van de Vijver, F.J.R., \& Leung, K. (1997). Methods and data analysis for cross-cultural research. Newbury Park, CA:Sage. 
Van de Vijver, F.J.R.,\& Leung, K. (2001). Personality in cultural context: Methodological Issues. Journal of Personality, 69,1007-1031.

van Ijzendoorn, M.H., Sagi, A. (1999). Cross-Cultural Patterns of Attachment: Universal and Contextual Dimensions. In J. Cassidy and P.R. Shaver (Eds), Handbook of attachment. New York: Guilford Press, 713-734.

Vereijken, C.J.L., Hanta, S. \& van Lieshout, C.F.M. (1997). Validity of attachment Qsort descriptions by mothers: the Japanese case. Japanese Psychological Research, 39, 291-299

Wang,Y. \& Ollendick, T.H. (2001). A Cross-Cultural and Development Analysis of SelfEsteem in Chinese and Western Children. Clinical Child and Family Psychology Review, 4, 253-271.

Waters, E. \& Cummings, E.M. (2000). A secure base from which to explore close relationships. Child Development ,71, 164-172.

Waters, H.S., Rodrigues, L. M., \& Ridgeway, D. (1998). Cognitive underpinnings of narrative attachment assessment. Journal of Experimental Child Psychology, 71, 211-234.

Yamada, A. (2001). Jyodo - ninchi no gokizoku to syori no junjyosei. Taijin shakai shirigaku kenkyu. [The misattribution and Primacy of Processing of Emotion and Cognition]. Japanese Journal of Interpersonal and Social Psychology, 1, 171-184.

Yang, B., Ollendick,T.H., Dong, Q., Xia, Y. \& Lin L. (1995). Only children and children with siblings in the People's Republic of China: Levels of Fear, Anxiety, and Depression. Child development, 66, 1301-1311.

Ye G., \& van Raaij, W.F. (1997). What inhibits the mere-exposure effect: Recollection or familiarity? Journal of Economic Psychology 2 18, 629-648.

Yonelinas, A.P. (2002). The nature of recollection and familiarity: A review of 30 years of research. Journal of Memory and Language, 46, 441-517.

Zajonc, R.B. (2001). Mere exposure: A gateway to the subliminal. Current Directions in Psychological Science, 10, 224-228. 